\title{
COVID-19 vaccine design: the Janus face of immune enhancement
}

Peter J. Hotez ${ }^{1,2,3,4 凶}$, David B. Corry ${ }^{5,6 凶}$ and Maria Elena Bottazzi (1) ${ }^{1,2,3 凶}$

Previous work on severe acute respiratory syndrome coronavirus (SARS-CoV) vaccines identified cellular immunopathology and antibody-dependent enhancement as potential safety issues. We discuss the implications of these findings for COVID-19 vaccine development and our approach to optimizing for safety and efficacy.

In the coming weeks and months, we might expect that at least a dozen new COVID-19 vaccines will enter clinical testing and development. These experimental vaccines comprise an array of both innovative and traditional technologies, ranging from packaged nucleic acids and virus vectors to recombinant proteins combined with adjuvants ${ }^{1}$. The design of these vaccines builds on human immunology data collected since 2003 when the severe acute respiratory syndrome coronavirus (SARS-CoV) first emerged in southern China, together with almost two decades of studies conducted in experimental animals and in vitro models.

Relevant population studies in humans include the evaluation of persistent antibodies against SARS-CoV in recovered patients, especially human antibodies directed to the SARS-CoV spike (S) protein and its receptor-binding domain (RBD), the component required for virus binding to its only known host cell entry receptor angiotensin-converting enzyme 2 $(\mathrm{ACE} 2)^{2}$. Because transferred plasma from patients who have recovered from SARS-CoV may reduce mortality, many investigators now pursue the $S$ protein or the RBD as vaccine targets. Indeed, studies conducted in a range of experimental animals, including transgenic mice expressing human ACE2, ferrets and non-human primates, confirm that such vaccines confer protective immunity against SARS-CoV challenge infections.

Although COVID-19 caused by SARS-CoV-2 has only recently emerged, there are already studies underway to examine whether the $\mathrm{S}$ protein or its RBD show similar promise as vaccine targets. Early clinical testing will require the rapid acceleration of new SARS-CoV-2 vaccines ${ }^{1}$, or in some cases repurposing of 'shovel-ready' vaccines already developed to the SARS-CoV counterparts ${ }^{3}$ based on findings that neutralizing antibodies to SARS-CoV can cross-bind and neutralize SARS-CoV-2 (REF. $\left.{ }^{4}\right)$.

While it is essential to advance COVID-19 vaccines in time to use them for this current pandemic, we must also recognize that there are potential safety issues that could slow the clinical development path and testing. Although two phase I clinical trials conducted previously for SARS vaccines have not revealed early safety issues, there are concerns based on observations made either in vitro or in experiments where animals received SARS-CoV vaccines. Specifically, those studies identify two potential safety signals in immunized animals following virus challenge: cellular immunopathology; and antibody-dependent enhancement (ADE). Here, we briefly summarize this dual or Janus-face of immune enhancement and offer our viewpoint on how this informs COVID-19 vaccine design.

\section{Cellular immunopathology}

During early testing of the first experimental SARS-CoV vaccines, following immunization and viral challenge infections, some experimental animals developed lung or liver histopathology characterized by significant tissue infiltration of lymphocytes, monocytes and eosinophils ${ }^{5}$. A predominance of eosinophils linked to tissue immunopathology prompted concerns that $\mathrm{T}$ helper $2\left(\mathrm{~T}_{\mathrm{H}} 2\right)$-type immune responses might be responsible, sometimes directed to virus-induced expression of the

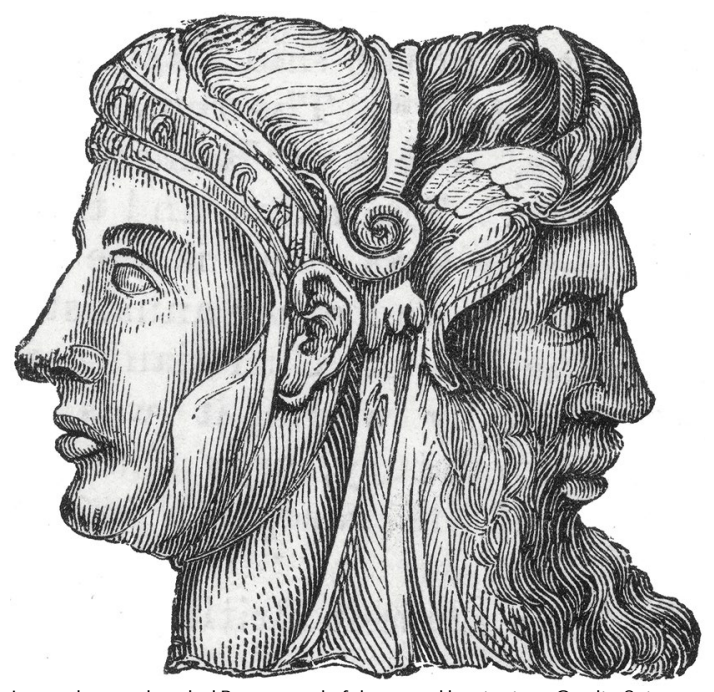
History Images/Alamy
Janus, the two-headed Roman god of doors and beginnings.Credit: Science 
SARS nucleocapsid (N) protein. However, our in-depth literature analysis suggests that $\mathrm{T}_{\mathrm{H}} 17$ responses may direct these cellular responses ${ }^{6}$, following immunization with inactivated viruses and vaccines delivered in virus vectors, and other key elements. In part, this evidence includes the link between $\mathrm{T}_{\mathrm{H}} 17$ cell development and IL-6, a cytokine strongly upregulated in patients with COVID-19 who experience cytokine storm (together with IL-8 induction). Further support comes from the role of IL-17 in promoting the activation, recruitment from bone marrow and extravasation of eosinophils into target organs, such as the lungs ${ }^{7}$, and the finding that alum, an adjuvant that promotes $\mathrm{T}_{\mathrm{H}}$ 2-type immunity, actually reduces immunopathology $y^{5}$. Such observations highlight the potential importance of selecting vaccine delivery platforms and adjuvants that shift host responses away from a $\mathrm{T}_{\mathrm{H}} 17$-type immune bias.

\section{Antibody-dependent enhancement}

$\mathrm{ADE}$ is a second concern and generally results when non-neutralizing antibodies bind to newly infecting virus to promote enhanced virus uptake into host cells via $\mathrm{Fc} \gamma$ receptors $(\mathrm{F} c \gamma \mathrm{Rs})^{8}$. Perhaps the best known example of ADE occurs following infection with multiple dengue virus serotypes, first reported by Halstead and O'Rourke in the 1970s and now influencing the design of new dengue vaccines. However, ADE may also influence the clinical course of several important human respiratory virus infections. For example, an observational study found that the 2008-2009 trivalent inactivated seasonal influenza vaccine might have caused enhanced disease during H1N1 pandemic flu, although another study actually found the opposite. Immune-enhanced disease resulting from the formalin-inactivated respiratory syncytial virus (RSV) vaccine in the 1960s may also partially result from ADE, possibly from non-neutralizing antibody to virus antigens that were altered by the formalin, although it is also possible that the $\mathrm{T}_{\mathrm{H}} 17$-type responses highlighted above might also be relevant.

There are conflicting data for the role of ADE in serious coronavirus infections. Antibodies against the $S$ protein can enhance virus uptake by cells in vitro, although the clinical relevance of these findings is conflicting. For example, there are clinical studies finding that SARS-CoV-specific antibodies are not harmful in patients with SARS, although it has been noted that non-neutralizing coronavirus antibodies may cause ADE in feline infectious peritonitis. Such efforts have prompted investigators to remove potential ADE-promoting $S$ protein epitopes located outside the $\mathrm{RBD}$ and focus on the RBD as a lead vaccine candidate ${ }^{9,10}$.

A vaccine using a protein-based SARS-CoV RBD is under development by our group to provide protective immunity against homologous virus challenge, while minimizing or preventing immune enhancement ${ }^{9,10}$. It is also being advanced as a potential heterologous vaccine against SARS-CoV-2 based on cross-binding and cross-neutralization studies using pseudoviruses, convalescent serum and polyclonal and monoclonal antibodies. Whether or not a heterologous vaccine might enhance the risk of ADE is unclear. For example, ADE has been noted in experimental animals vaccinated with heterologous inactivated viruses, possibly due to non-neutralizing antibodies. However, from studies using a MERS-CoV vaccine, it has also been proposed that neutralizing antibodies might instead induce ADE.

\section{Concluding comments}

How does the discussion above inform vaccine design, development and testing? Among the major findings of highest consideration are, one, preclinical testing in laboratory animal virus challenge models finds that experimental vaccines in virus vectors can cause immunopathology owing to mononuclear cell and eosinophil infiltration of the lung. Two, conversely, alum reduces cellular infiltration, while $\mathrm{ADE}$ in vitro is linked to both non-neutralizing antibodies outside the $S$ protein $\mathrm{RBD}$, as well as neutralizing antibodies. While we do not have strong evidence that such in vitro studies or animal challenge models are predictive of clinical safety, they point to the promise of subunit vaccines including the RBD and alum adjuvants. We are now working to advance this approach into the clinic for phase I studies.

1. Thanh Le, T. et al. The COVID-19 vaccine development landscape. Nat. Rev. Drug Discov. https://doi.org/10.1038/d41573-02000073-5 (2020)

2. Cao, Z. et al. Potent and persistent antibody responses against the receptor-binding domain of SARS-CoV spike protein in recovered patients. Virol. J. 7, 299 (2010).

3. Hotez, P. J. \& Bottazzi, M. E. Developing a low-cost and accessible COVID-19 vaccine for global health. Preprints 2020, 2020030464 (2020)

4. Tai, W. et al. Characterization of the receptor-binding domain (RBD) of 2019 novel coronavirus: implication for development of RBD protein as a viral attachment inhibitor and vaccine. Cell. Mol. Immunol. https://doi.org/10.1038/s41423-020-0400-4 (2020).

5. Tseng, C.-T. et al. Immunization with SARS coronavirus vaccines leads to pulmonary immunopathology on challenge with the SARS virus. PLoS One 7, e35421 (2012).

6. Hotez, P. J., Bottazzi, M. E. \& Corry, D. B. The potential role of $\mathrm{T}_{H} 17$ immune responses in coronavirus immunopathology and vaccineinduced immune enhancement. Microbes Infect. https://doi.org/ 10.1016/j.micinf.2020.04.005 (2020).

7. Keely, S. \& Foster, P. S. Stop press: eosinophils drafted to join the Th17 team. Immunity 43, 7-9 (2015).

8. Smatti, M. K. Al Thani, A. A \& Yassine H. M. Viral-induced enhanced disease illness. Front. Microbiol. 9, 2991 (2018).

9. Du, L. et al. Receptor-binding domain of SARS-CoV spike protein induces long-term protective immunity in an animal model. Vaccine 25, 2832-2838 (2007).

10. Chen, W. H. et al. Optimization of the production process and characterization of the yeast-expressed SARS-CoV recombinant receptor-binding domain (RBD219-N1), a SARS vaccine candidate. J. Pharm. Sci. 106, 1961-1970 (2017).

\section{Acknowledgements}

Owing to space and other limitations the authors wish to acknowledge the many authors who contributed primary research papers contributing to this effort who are not cited here.

Author contributions

The authors contributed equally to all aspects of the article.

\section{Competing interests}

P.J.H. and M.E.B. are investigators leading the development of coronavirus vaccines against SARS-CoV, MERS-CoV and SARS-CoV-2. D.B.C. is a scientific advisor and holds intellectual property in Atropos Therapeutics, LLC. 